\title{
Large Eddy Simulation of the Flow Past a Circular Cylinder
}

\author{
You Yi ${ }^{1,2, a}$, Yan Zhitao ${ }^{3,1, b}$, Zhong Yongli ${ }^{1, c}$, Li Yongjie ${ }^{2}$ \\ (1.School of Civil Engineering, Chongqing University, Chongqing 400045, China;
}

2. State Grid Xinjiang Electric Power Company Electric Power Research Institute, Urumqi

830011 , China ;

\begin{abstract}
3. School of Civil Engineering, Chongqing University of Science \& technology, Chongqing 401331, China)
\end{abstract}

a97080463@qq.com, byanzhitao@cqu.edu.cn, czhongyongli@cqu.edu.cn

Key words: numerical simulation; flow around a cylinder; large eddy simulation; lift coefficient

Abstract: The numerical simulation method was used to investigate the flow past a single cylinder. The flow field structure and the characteristic parameters of the cylindrical surface were analyzed by large eddy simulation. The numerical results show that the calculation of the length of the recirculation zone for computation of the mean velocity in the cylinder center line direction is slightly larger than the experimental results. Both the steady pressure coefficient and wake vortex shedding frequencies are coincide with the experimental data. The large eddy simulation for flow past a cylinder has high accuracy. The effect of Reynolds number and surface roughness is simulated based on the model of large eddy simulation, and the results show that the drag coefficient is obviously affected by the equivalent roughness.

\section{Introduction}

The turbulent flow past a circular cylinder exists extensively in engineering application and is a very classical problem of fluid mechanics ${ }^{[1,2,3]}$. Von Kama ${ }^{[4]}$ determine the relationship between wake flow resistance and vortex system momentum by the analysis of the separation vortex system behind cylinder. Thereafter researchers had done a lot of theoretical and experimental studies on this phenomenon and a lot of valuable experimental data was produced. Cantwell ${ }^{[5]}$ carried out an experimental study on fluid flow around a smooth single cylinder and the cylindrical surface load characteristics and flow field distribution are provided in detail. Wang-Yaling ${ }^{[6]}$ numerically simulated a uniform viscous and incompressible flow around a circular cylinder by the method of computational fluid dynamics. The results showed that the flow around a circular cylinder with high Reynolds number has obvious three-dimensional characteristics. Yao-Xiongliang ${ }^{[7]}$ achieved the same conclusion by using Large-eddy simulation method to calculate the hydrodynamic characteristics of a rigid single circular cylinder in three dimensional incompressible uniform cross flow.

The aerodynamic forces generated by the vortex shedding on both sides of the structure is likely to cause structural damage in wind engineering. In this paper, the large eddy simulation(LES) is used to simulate the flow around a cylinder. The effect of Reynolds number on the drag coefficient, $C_{d}$ is studied under the condition of smooth and equivalent roughness. The numerical results are then compared to the available data in the literature to carry out a series of comprehensive analysis of the flow around the cylinder. The conclusion can provide some guidance for structural wind-resistant design. 


\section{Computational methodology}

As the turbulent motion is composed of vortices of different size, large eddy simulation uses a spatial filtering operation to separate the larger and smaller eddies. The former is directly computed and the latter is modeled by subgrid-scale ${ }^{[8]}$. The governing equations for a viscous incompressible fluid flow are as follow ${ }^{[9]}$ :

$$
\begin{aligned}
& \frac{\mathrm{D} \rho}{\mathrm{D} t}+\nabla \cdot \rho U=0 \\
& \frac{D u_{i}}{D t}=f_{i}+\frac{1}{\rho} \sum_{j=1}^{3} \frac{\partial \sigma_{i j}}{x_{j}}(i=1,2,3)
\end{aligned}
$$

Where $\rho$ is the density of air ; $u_{i}(i=1,2,3)$ are the mean velocity in $x\left(x_{1}\right), y\left(x_{2}\right), z\left(x_{3}\right)$ direction, respectively ; $f_{i}$ is the component of the force acting on the fluid per Unit volume, $\sigma_{\mathrm{ij}}$ is the internal stress per unit volume,$\nabla$ is the Hamiltonian and can be written as:

$$
\nabla=i \frac{\partial}{\partial x}+j \frac{\partial}{\partial y}+k \frac{\partial}{\partial z}=e_{i} \frac{\partial}{\partial x_{i}}
$$

The computational domain is show in Fig. 1(a), The coordinate origin locate at the center of the bottom of cylinder. The $x$ axis parallel to the streamwise direction and the $y$ axis parallel to the spanwise direction. The distance from the cylinder to the inlet is $5 D$, where $D=57 \mathrm{~mm}$, is the Cylinder diameter. The Reynolds number based on cylinder is 7000. At the inflow plane constant velocity is imposed; at the exit plane, a pressure outlet boundary condition is applied; No-slip wall boundary condition is used for cylindrical surface. The other boundary conditions are specified as Symmetry at the top, bottom, left and right planes of the computational domain. Previous large eddy simulation studies have shown that Smagorinsky-Lilly model is appropriate when Computer performance is not enough ${ }^{[10.11,12]}$. The time is $5 \mathrm{e}^{-4}$ based on the principle that Courant is number less than 1. The first grid point of the cylindrical surface is at $y^{+}<1$.

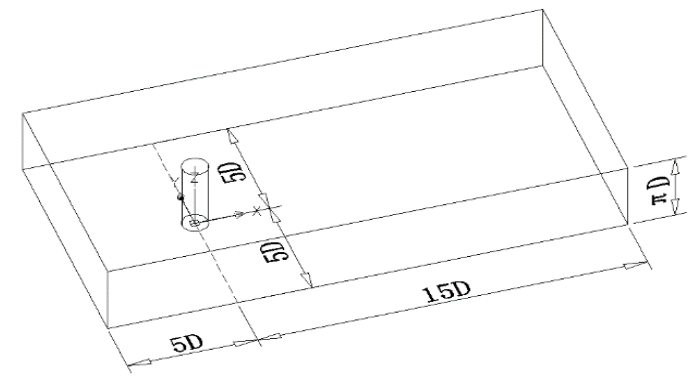

(a) Computational domain

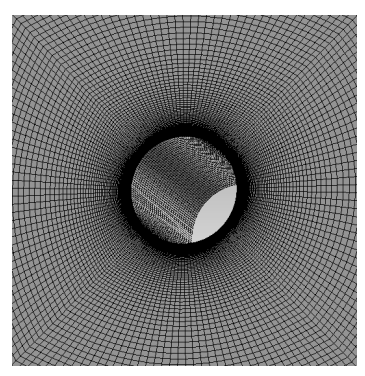

(b) The mesh near the cylinder

Fig. 1 The computational domain and meshing

\section{Results and discussion}

The characteristics of flow field 
Fig. 2 is the time-averaged streamwise velocity distribution at all centerline stations downstream of the cylinder. The recirculation zone length of calculation, $l_{\mathrm{R}} / D=0.52$, i.e. the curve length where the time-averaged velocity is negative. Minimum reflux velocity normalized by the inlet velocity is $U / U_{0}=-0.196$. Comparing with the experimental results of Cantwell \& Coles (1983), reflux velocity region is slightly larger and the wake recovered faster.

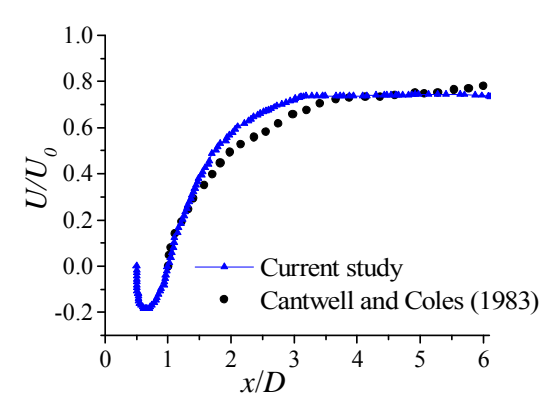

Fig. 2 The time average velocity distribution of $\mathrm{x}$ direction along the cylindrical center

The mean velocity distribution at $x=D$ is shown in Fig. 3. It can be seen that the mean velocity in this section is $V$ type. The cylinder only affects the flow field near it, in the range of $-D$ and $D$. The calculated results are in good agreement with the experimental data except that the minimum mean velocity $U / U_{0}=-0.08$, is smaller than experiment. It is shown that the velocity at $x / D=1$ of the center line of the cylinder is less than 0 and still in the recirculation zone. This is exactly the same as the result of Fig. 2.

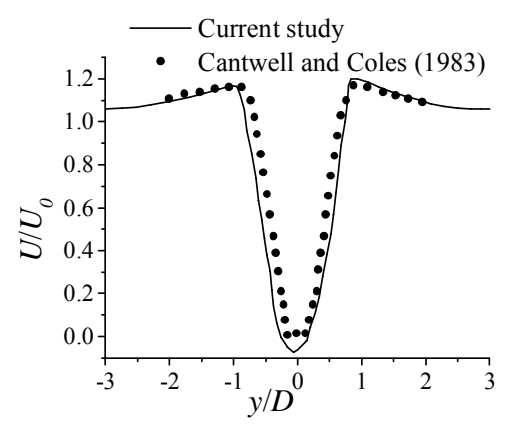

Fig. 3 The time average velocity distribution of $\mathrm{x} / \mathrm{D}=1$ cross section

Fig. 4 is the instantaneous velocity contours on the section of $y / D=0$ at time of $t=5.0 \mathrm{~s}$, where the dotted line represents a negative velocity. In Fig.4 $(a)$ it can be found that in the region of about $D$ behind the cylinder, there exists an obvious recirculation zone. In Fig. $4(b)$, the positive and negative regions of spanwise velocity appear alternately, which indicates the formation of Karman Vortex Street. The fact that the zero line of velocity (the intersection of the positive and negative velocity region) is not parallel to the cylinder has also shown the existence of the three-dimensional effect. Fig. $4(c)$ is the distribution of the instantaneous wall-normal velocity. In the far wake region, there still exist obvious vortices, which show that the numerical dissipation of the large eddy simulation is very small and the numerical simulation is accurate. 


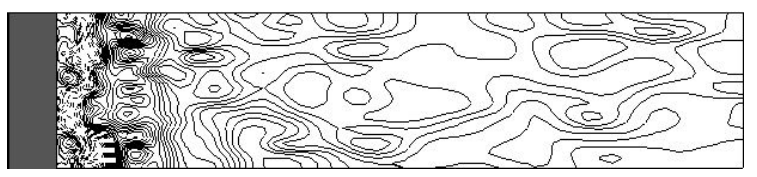

(a) X velocity

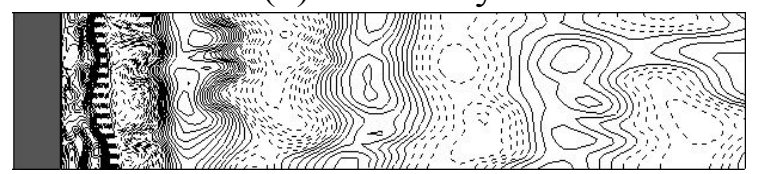

(b) Y velocity

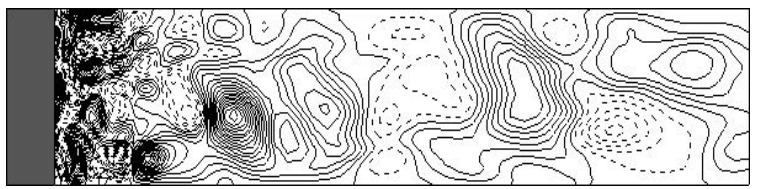

(c) Z velocity

Fig. 4 The time average velocity distribution of $y / D=0$ cross section, $t=5.0 \mathrm{~s}$

Fig. 5 is the vorticity contours of the lift coefficient $C_{L}$ at different times within one period. It can be seen from the Fig. that the wake behind the cylinder has the vortex of alternating shedding from top to bottom, and the vortex has no definite structure form and moving route, which shows more randomness. The vortex moves backward over time, and it can be seen that the area of the vortex becomes smaller because of the dissipation of the vortex. The turbulent irregular vortex street has formed. The vortex shedding is consistent with the characteristics of the flow around a single cylinder in the subcritical Reynolds number region.

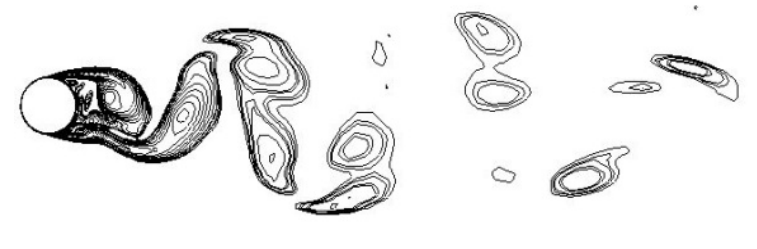

(a) $t=T / 4$

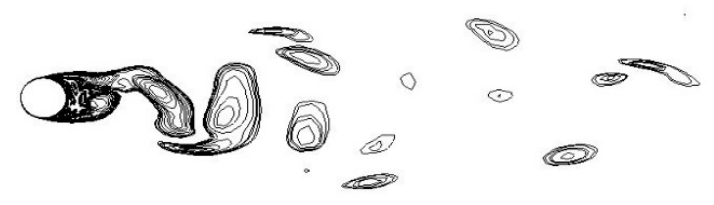

(c) $t=3 T / 4$

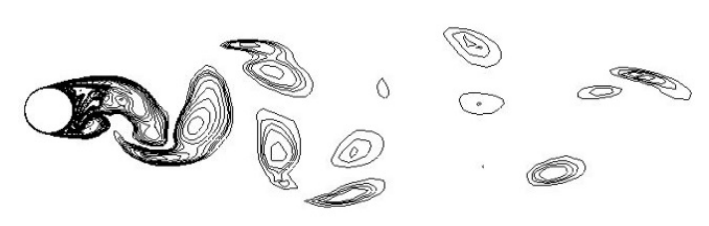

(b) $t=T / 2$

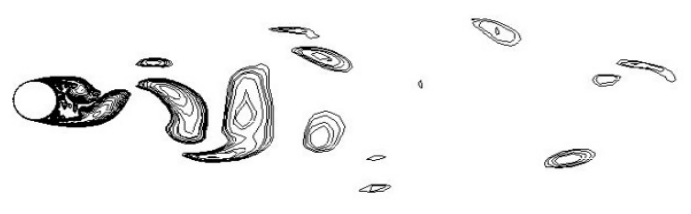

(d) $t=T$

Fig. 5 The vorticity graph within one period of a lift coefficient

3.2 Steady pressure coefficient

The pressure distribution on the cylinder surface can be expressed by the pressure coefficient $C_{p}:$

$$
C_{P}=\frac{P-P_{0}}{\frac{1}{2} \rho U_{0}^{2}}
$$

Where $P$ is Static pressure on cylindrical surface; $P_{0}=0 \mathrm{~atm}$ is reference pressure and $\rho$ is the density of air. 


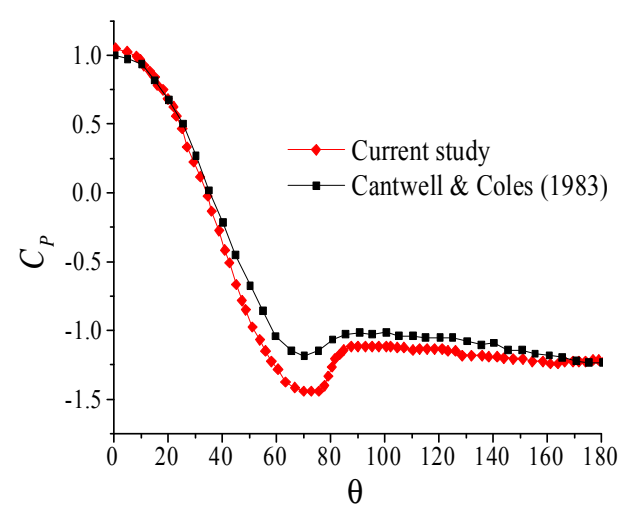

Fig. 6 Pressure coefficient distribution With cylindrical circumferential

Fig.6 shows the distribution of time averaged steady pressure coefficient $C_{P}$ along the circumference. Only the distribution curve in the range of $0 \sim 180$ degrees is given because of the symmetry of the pressure distribution. As can be seen from the Fig.6, the maximum pressure coefficient on the surface of the cylinder is 1 . The pressure coefficient decreases while the flow velocity increases gradually and then after reaching the minimum value, it returns to a relatively stable value and forms a flat steady-state pressure distribution behind the cylinder. This is in agreement with the experimental results. The minimum pressure angle is $78^{\circ}$, i.e., the separation angle, which is slightly larger than the experimental value of $75^{\circ}$, and the maximum negative pressure is slightly larger than the experimental results.

3.3 The aerodynamic coefficients and the Strouhal number

The time history of the drag coefficient $C_{D}$ and the lift coefficient $C_{L}$ of the t=4.9 5.0s period is extracted from the results of numerical simulation, as shown in Fig. 7. In the first period of $0.1 \mathrm{~s}$, the $C_{D}$ fluctuates through about 26.5 cycles, while the $C_{L}$ fluctuates through about 13.3 cycles.

The frequency of $C_{D}$ and $C_{L}$ variation can be defined as:

$$
\begin{aligned}
& f_{D}=\frac{1}{T_{D}} \\
& f_{L}=\frac{1}{T_{L}}
\end{aligned}
$$

Where $T_{D}$ and $T_{L}$ are the cycle of $C_{D}$ and $C_{L}$, respectively, then:

$$
f_{D} \approx 2 f_{L}
$$

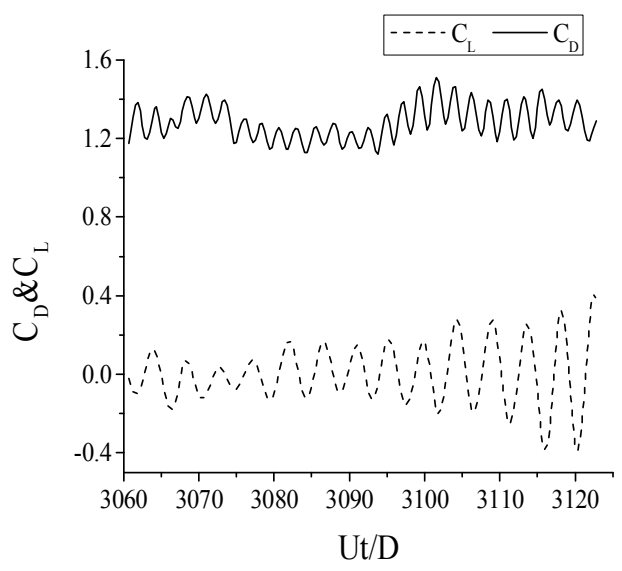

Fig. 7 The change of drag coefficient and lift coefficient with time 
Cylindrical wake vortex shedding frequency can be expressed by a dimensionless parameter ${ }^{[13]}$, it is called the Strouhal number St, defined as:

$$
S t=\frac{D f}{U_{0}}
$$

In current study it has the value $S t \approx 0.214$ and is in good agreement with the experimental value of Simiu(1992) .

\subsection{Reynolds number Effect}

Wieselsberger ${ }^{[14]}$ provided the complete curve of friction coefficient with the Reynolds number in the range of $10^{-1} \sim 10^{6}$ by experimental method. The influence of Reynolds number on the drag coefficient is studied numerically in this paper. As can be seen from Fig.8, the calculated results are in good agreement with the experimental results. The drag coefficient of the cylinder in the subcritical region does not enter the descending section, and the drag coefficient is always nearly 1.2 .

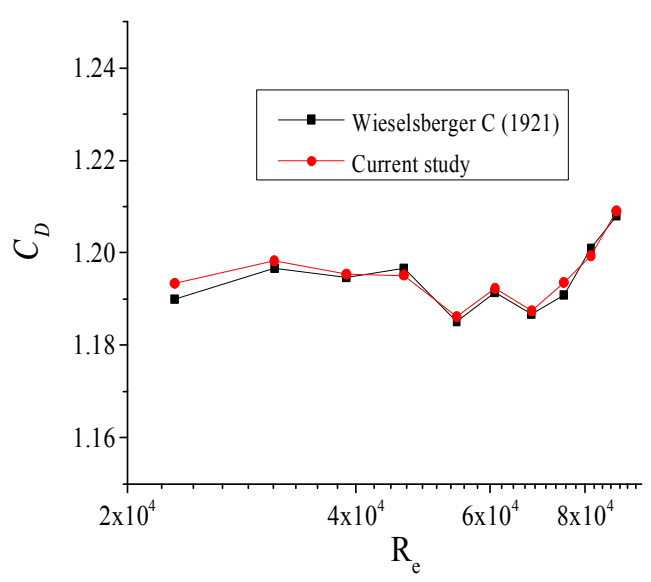

Fig. 8 The comparison between smooth cylindrical test results and reference value

\subsection{Effect of surface roughness}

The influence of different surface roughness values on the mechanical properties of the single cylinder is analyzed. Because the single cylinder model is completely symmetrical in geometry, the lift coefficient $C_{L}$ is very small, and the torsion coefficient $C_{M}$ is negligible. In general, the Reynolds number effect is mainly concerned with the relationship between the drag coefficient and Reynolds number. Therefore, the influence of the equivalent roughness of the cylinder surface on the drag coefficient is mainly investigated in current study.

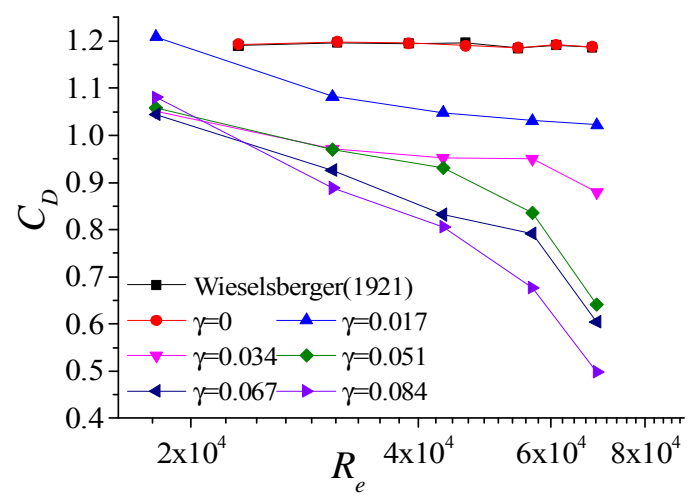

Fig. 9 The drag coefficient of different equivalent roughness 
Fig. 9 is the drag coefficient with different equivalent roughness. The drag coefficient curve is obviously different. Compared with the smooth cylinder, the drag coefficient curve began to decline when the equivalent roughness is 0.017 . The decrease of drag coefficient curve is more complete and the same as that of critical Reynolds number. When $\gamma$ continues to increase to 0.051 and greater, the drag coefficient curve has a complete descending segment and this is consistent with drag coefficient of the supercritical Reynolds number.

\section{Conclusions}

Numerical simulation of flow past a cylinder at high Reynolds number is carried out by large eddy simulation. The results show that prediction of the mean streamwise velocity of the central line of the cylinder is slightly larger than the experimental data. The velocity distribution at $x=D$ behind the cylinder has a $V$ profile. The wake vortex moves backward as time goes on; the area of vortex becomes smaller due to dissipation and the turbulent irregular vortex street has formed. The time averaged steady pressure coefficient $C_{P}$ and the shedding frequency of the wake vortex region are in good agreement with the experimental results. Large eddy simulation has high accuracy for the simulation of flow past a cylinder. The effects of Reynolds number and surface roughness are further investigated. The results show that the equivalent roughness has a significant influence on the drag coefficient.

\section{Acknowledgements}

This work was supported by the National Natural Science Funding (China, 51478069).

\section{References}

[1] Simiu E, Scanlan R H. Wind effects on structure-An introduction to wind engineering[M]. New York: John Wiley \& Sons, Inc, 1992.

[2] Anderson.J.D. Fundamentals of Aerodynamics (Fourth edition)[M]. New York: McGraw-Hill Companies, Inc, 2011

[3] Xia Xueyu. Engineering separation flow dynamics [M].Beijing: Beihang University Press, 1991.

[4] VON KARMN TH, RUBACH H .On the mechanism of resistance in fluid[J].Physikalis che Zeitschrift , 1912,13 (5):351-358 .

[5] Cantwell B, Coles D. An experimental study of entrainment and transport in the turbulent near wake of a circular cylinder [J]. Journal of Fluid Mechanics, 1983, 139: 321-374.

[6] Wang Yaling, Liu Yingzhong, Miao Guoping. Three dimensional numerical simulation of viscous flow around circular cylinder [J]. Journal of Shanghai Jiaotong University, 2001, 35(19):1464-1469.

[7] Yao Xiongliang, Fang Yuanyuan. Three dimensional numerical simulation of the flow past a circular cylinder based on LES method [M]. Journal of hydrodynamics Ser A, 2007, 22 (5)564-572.

[8] D. C. Wilcox. Turbulence Modeling for CFD (Third Edition). DCW Industries, Inc. La Canada, California, 2006..

[9] H.K. Versteeg; W. Malalasekera. An Introduction to Computational Fluid Dynamics: The Finite Volume Method (2nd Edition). Pearson Education. Harlow, England, 2007.

[10] Beaudan, P., Moin, P., 1994. Numerical experiments on the flow past a circular cylinder at sub-critical Reynolds number. Report No. TF-62, Department of Mechanical Engineering. 
Stanford University.

[11] Breuer, M., 1998. Large eddy simulation of the subcritical flow past a circular cylinder: numerical and modeling aspects. International Journal for Numerical Methods in Fluids. 28:1281-1302.

[12] Kravchenko, A.G., Moin, P., 2000. Numerical studies of flow over a circular cylinder at $\mathrm{Re}_{\mathrm{D}}=3900$. Physics of Fluids. 12(2):403-417.

[13] H.Schlichting, K.Gersten. Boundary layer theory (8thEdition). Springer-Verlag , Berlin Heidelberg, New York, 2003.

[14] Wieselsberger C. Neuere festellungen ber die Gesetze des Flssigkeits und Luftwider-stands [J]. Physikalische Zeitschrift, 1921, 22(3): 321-328 